\title{
La ponctuation dans le texte : parenthèses, sujets et linéarité dans l'incipit du Palace de Claude Simon
}

Sabine Boucheron

\section{(2) OpenEdition Journals}

Édition électronique

URL : http://journals.openedition.org/rlv/1212

DOI : 10.4000/rlv.1212

ISSN : 1958-9239

Éditeur

Presses universitaires de Vincennes

\section{Édition imprimée}

Date de publication : 1 mai 1999

Pagination : $33-40$

ISSN : 0986-6124

\section{Référence électronique}

Sabine Boucheron, «La ponctuation dans le texte : parenthèses, sujets et linéarité dans l'incipit du Palace de Claude Simon », Recherches linguistiques de Vincennes [En ligne], 28 | 1999, mis en ligne le 09 septembre 2005, consulté le 19 avril 2019. URL : http://journals.openedition.org/rlv/1212 ; DOI : 10.4000/rlv. 1212 


\title{
Sabine BOUCHERON \\ Université de la Sorbonne nouvelle - Paris 3 RES \\ (SYLED-UPRES, EA 2290)
}

\section{LAPONCTUATION DANS LE TEXTE : PARENTHÈSES, SUJETS ET LINÉARITÉ DANS L'INCIPIT DU PALACE DE CLAUDE SIMON}

\begin{abstract}
RÉSUMÉ
Nous proposons d'aborder la problématique de la linéarité par le biais de la ponctuation, et particulièrement grâce à la parenthèse. Dans l'incipit du Palace de Claude Simon, on peut en effet observer que la linéarité langagière se dédouble. Un premier niveau, qui obéit aux normes scripturaires du nouveau roman, offre une description hyperréaliste d'un pigeon ; un second niveau, ménagé par les parenthèses, réintroduit des traces de subjectivité : des personnages de roman, la subjectivité modalisante d'un narrateur et le fil de l'écriture qui revient au cœur du texte. Un texte qui s'écrit donc sur plusieurs lignes, et qui permet de laisser croire qu'il est enfin possible d'échapper à cette linéarité langagière qui, d'ordinaire, contraint l'écrivain.
\end{abstract}

\section{MoTS-CLÉS}

Parenthèse, ponctuation, texte, linéarité, subjectivité, dédoublement, décrochement (typo)graphique. 
C'est dans le cadre d'une recherche de doctorat sur les signes d'insertion que sont la parenthèse et le tiret double que nous est apparue la nécessité d'une approche particulière de la linéarité. Plus précisément, c'est dans la toute première phrase du Palace de Claude Simon que nous proposons d'analyser les enjeux - et les jeux - de la linéarité langagière avec ce que nous appelons l'opération de décrochement typographique, c'est-à-dire la mise entre parenthèses ou entre tirets d'un ou de plusieurs segments ${ }^{1}$.

Et à un moment, dans un brusque froissement d'air aussitôt figé (de sorte qu'il fut là - les ailes déjà repliées, parfaitement immobile - sans qu'ils l'aient vu arriver, comme s'il avait non pas volé jusqu'au balcon mais était subitement apparu, matérialisé par la baguette d'un prestidigitateur), l'un d'eux vint s'abattre sur l'appui de pierre, énorme (sans doute parce qu'on les voit toujours de loin), étrangement lourd (comme un pigeon en porcelaine, pensa$\mathrm{t}$-il, se demandant comment dans une ville où la préoccupation de tous était de trouver à manger ils s'arrangeaient pour être aussi gras, et aussi comment il se faisait qu'on ne les attrapât pas pour les faire cuire), avec son soyeux plumage tacheté, gris foncé, à reflets émeraude sur la nuque et cuivrés sur le poitrail, ses pattes corail, son bec en forme de virgule, sa gorge bombée : quelques instants il resta là, l'œil stupide et rond, tournant la tête sans raison à droite et à gauche, passant d'une position à l'autre par une série de minuscules et brefs mouvements, puis (sans doute parce que l'un de ceux qui étaient dans la chambre fit un geste, ou un bruit), aussi brusquement qu'il s'était posé, il s'envola.

Phrase complexe, où l'opération de décrochement typographique dédouble la linéarité langagière en un double fond qu'il faut reconstituer ainsi ${ }^{2}$ :

Et à un moment, dans un brusque froissement d'air aussitôt figé

(de sorte qu'il fut là

- les ailes déjà repliées, parfaitement immobile -

sans qu'ils l'aient vu arriver, comme s'il avait non pas volé jusqu'au balcon mais était subitement apparu, matérialisé par la baguette d'un prestidigitateur),

l'un d'eux vint s'abattre sur l'appui de pierre, énorme

étrangement lourd

(sans doute parce qu'on les voit toujours de loin), 
(comme un pigeon en porcelaine, pensa-t-il, se demandant comment dans une ville où la préoccupation de tous était de trouver à manger ils s'arrangeaient pour être aussi gras, et aussi comment il se faisait qu'on ne les attrapât pas pour les faire cuire),

avec son soyeux plumage tacheté, gris foncé, à reflets émeraude sur la nuque et cuivrés sur le poitrail, ses pattes corail, son bec en forme de virgule, sa gorge bombée: quelques instants il resta là, l'œil stupide et rond, tournant la tête sans raison à droite et à gauche, passant d'une position à l'autre par une série de minuscules et brefs mouvements, puis

(sans doute parce que l'un de ceux qui étaient dans la chambre fit un geste, ou un bruit),

aussi brusquement qu'il s'était posé, il s'envola.

Ainsi « purifiée » des parenthèses qui viennent en perturber le cours, la phrase insérante peut se lire aisément. Il s'agit alors d'une description froide et objective, sans point de vue, celle d'un pigeon qui se pose sur un balcon. Cet espace insérant forme un premier niveau linéaire, celui de l'objectivité pure ; cependant, les parenthèses qui le surchargent permettent l'insertion d'un second niveau linéaire. La première parenthèse se situe entre les deux circonstants initiaux et le sujet grammatical de la phrase insérante («l'un d'eux »). Le premier pronom contenu dans la parenthèse («il ») désigne le pigeon - au cœur de l'événement. On peut noter que cette première parenthèse, qui vient se greffer sur la phrase insérante, se creuse à son tour pour laisser place à un nouvel élément inséré par un double tiret (« - les ailes déjà repliées, parfaitement immobile - »). Cet enchâssement des éléments insérés permet à la fois de multiplier les sujets grammaticaux et d'échapper à la stricte linéarité de l'écriture ; ou du moins, d'en donner l'illusion. Nous avons choisi de représenter le segment inséré par les tirets (qui concerne le pigeon) dans la première colonne, car il nous semble participer de la description objective : ainsi, le texte se dédouble et l'insertion dans l'insertion fait revenir au point de départ : celui du « miroir promené le long d'un chemin ».

La seconde parenthèse est insérée entre deux groupes adjectivaux ( « énorme » et « étrangement lourd») qualifiant « l'appui de pierre ». Cette seconde parenthèse est à la fois le « porte-voix » du narrateur, dont la présence apparaît dans l'adverbe de modalisation «sans doute », et le lieu d'apparition d'un nouveau sujet. «On », indéfini - s'agit-il du narrateur seul ? du lecteur ? des deux à la fois ?

Dans la troisième parenthèse, le narrateur donne à voir non plus sa propre subjectivité (« sans doute ») mais celle d'un « il », nouveau protagoniste, dont le discours intérieur est dévoilé : « se demandant comment». Non seulement la parenthèse brise la surface plane de la description, non seulement elle réintroduit 
des sujets indistincts, mais elle donne, pour ainsi dire, la parole au personnage renaissant de l'histoire, même s'il ne s'agit pour l'instant que d'un «il » anonyme.

La dernière parenthèse : « sans doute parce que l'un de ceux qui étaient dans la chambre fit un geste, ou du bruit » replace le récit du côté du narrateur, saisi dans sa subjectivité non omnisciente (« sans doute» apparaît pour la seconde fois).

Ce que nous appelons "l'espace du décroché », c'est-à-dire le site phrastique compris dans un signe double, entre un signe ouvrant et un signe fermant (ici, la parenthèse), apparaît, dans cette première phase du texte, comme le lieu d'un retour du sujet : sujet grammatical (les pronoms), sujet de l'histoire (les personnages), sujet écrivant - encore opaque - qui se donne à lire. Ce retour du sujet nous semble s'opérer par un dédoublement de la linéarité. Le niveau insérant permet une description parfaitement neutre du pigeon (respectant par là même l'objectivité radicale du nouveau roman) tout en acceptant - grâce à la greffe des parenthèses - une réintégration des sujets : sujets grammaticaux et acteurs d'une histoire, sujet écrivant sur une linéarité dédoublée. La parenthèse joue donc ici un rôle important : c'est en effet par elle que le personnage et l'écrivain reviennent au roman. Ce n'est évidemment pas une redécouverte du personnage classique, du héros réaliste; au contraire, ce qui se profile ici, c'est un sujet frappé d'indistinction : «Il», «Ils », « On », «Un », « Ceux ». Ainsi, tandis que la phrase insérante met en scène la description de l'oiseau, la parenthèse est le site énonciatif du sujet, du personnage du roman dont on saisit le discours indirect : « se demandant », mais aussi du narrateur (et de l'auteur ?). C'est en effet dans ce site de la phrase qu'il apparaît, notamment avec la comparaison « comme un pigeon en porcelaine » et à travers la modalisation : « sans doute». Cette première phrase expose les règles d'un dispositif qui sera constant tout au long du texte, allant même en se complexifiant.

Cette bipartition du texte : d'une part le texte insérant réaliste et purement descriptif et d'autre part le texte inséré où les sujets se démultiplient est le lieu de refuge d'un narrateur qui rencontre à la fois la multiplicité des voix («Il », «Ils », « On », « Un », « Ceux ») et la dualité des voies (ce que l'on pourrait appeler un dédoublement du linéaire). Ce constat nous conduit donc très précisément vers une autre interrogation - si chère aux yeux de Claude Simon -, celle du temps et de la linéarité ${ }^{3}$. C'est ainsi que Claude Simon déclarait : « [...] forcé par la configuration linéaire de la langue d'énumérer les unes après les autres les composantes de ce paysage [...], l'écrivain, dès qu'il commence à tracer un mot sur le papier, touche aussitôt à ce prodigieux ensemble, ce prodigieux réseau de rapports établis dans et par cette langue qui, comme on l'a dit "parle déjà avant nous" $[\ldots]^{4}$.»

Ces problématiques sont au cœur même de la réflexion menée par Jacqueline Authier (1993) ${ }^{5}$. "Cette linéarité, syntaxiquement réglée, selon laquelle tout énoncé se déroule vers sa fin, apparaît comme une pressante 
métaphore du temps dans lequel nous sommes pris ${ }^{6}$. » L'accumulation - le foisonnement - des parenthèses dans cette première phrase du Palace impose au texte un rythme ralenti par le déploiement de chaque élément inséré. En effet, la phrase insérante est contrariée - dans sa progression syntaxique - par la présence des parenthèses. La première de celles-ci, en particulier, placée comme nous l'avons vu, après deux circonstants initiaux, retarde d'autant l'apparition du prédicat : «L'un d'eux vint s'abattre. » Il en va de même pour la deuxième parenthèse qui intervient entre deux adjectifs coordonnés par une virgule. Les deux dernières parenthèses contribuent également à ce ralentissement du rythme de la phrase d'accueil ; la dernière étant enclavée entre deux circonstants antéposés au verbe "il s'envola ». Cet enchâssement de parenthèses permet donc de ralentir ou plutôt d'engourdir la progression syntaxique de la phrase insérante.

Ainsi « sur le mode subjectif et illusoire, c'est le temps lui-même, dont le déroulement syntaxique est l'image, qui est ralenti, retenu, suspendu, dans ces décrochements ${ }^{7}$ ». Cet « alanguissement » du déroulement syntaxique s'accompagne sans doute d'un fantasme de « délinéarisation ». De ce que J.Authier appelle un « hors-fil du cours syntaxique ${ }^{8} »$. Dans cette représentation fantasmatique de l'énoncé, on pourrait voir un premier fil - celui de la phrase insérante - auquel viendrait s'ajouter un second fil - celui de l'espace d'insertion. Quelle hiérarchie établir alors entre ces deux fils ? On serait tenté d'attribuer au second une position de « surplomb ${ }^{9}$ », tentation que la présence, à deux reprises, de la modalisation - « sans doute » - contribue à confirmer. Il y a dans ces « lignes »- cela « saute aux yeux »- un «travail » du linéaire et une aspiration vers un espace d'écriture pluridimensionnel et qui échapperait à l'impératif de la ligne unique.

Le texte se fantasme donc aux confins de l'écriture musicale ou de l'art pictural : pouvoir enfin écrire sur plusieurs lignes. Mais faire l'expérience de l'écriture, c'est « être contraint de présenter les uns après les autres des éléments qui dans la réalité se superposent [...]. Quand j'écris je suis forcé de plier ce magma de sensations, de souvenirs, de pensées [...] qu'il y a en moi simultanément [...], il faut bien que je les aligne dans un ordre puisque l'écriture est linéaire [...]. Les peintres ont bien de la chance ${ }^{10} »$. Ainsi, dans cette « tension des deux portées ${ }^{11}$ », le narrateur n'use pas de l'espace parenthétique pour prendre la parole, mais pour la perdre, ou plutôt pour commencer de la rendre aux personnages de l'histoire. Vertige des sujets. Et dans ce creusement du texte, dans cette superposition des niveaux descriptifs et subjectifs, ce qui est à l'œuvre, c'est bien la performance du sujet écrivant.

Sujet écrivant, sujet surplombant, sujet maître - ou illusoirement défini comme tel ? - des strates phrastiques, Claude Simon nous engage vers une définition de la parenthèse, des tirets et plus largement de la ponctuation comme un système dont l'autonomie par rapport à l'oral est proprement constitutive. Une 
lecture «à haute voix» ne rendrait pas l'effet de strates, de creusement du linéaire et de suspension du temps. Dégagée de l'oral et pour ainsi dire illisible à haute voix tant les espaces décrochés sont longs et imbriqués, la première phrase du Palace nous engage vers une définition de la ponctuation, ou du moins de certains de ses signes, comme les outils d'une écriture d'intérieur ou plutôt de l'intérieur, c'est-à-dire d'une écriture en marge de cet extérieur sur lequel donne à voir la lecture à haute voix.

Toutes les phrases du Palace contiennent une parenthèse en moyenne deux fois supérieure à la phrase insérante, ce qui implique une gymnastique à la fois oculaire et intellective à laquelle on doit se résoudre et qui constitue la marque de l'écriture simonienne. Dans le cadre d'une approche de la ponctuation résolument tournée vers l'écrit, l'espace du décroché, l'entre parenthèses, peut être défini comme l'indice de préoccupations et, pour ainsi dire, comme la marque visible d'inquiétudes multiples et pourtant propres à chaque sujet au moment même d'écrire : la variante toujours possible si l'on écrit sur deux lignes, et surtout le choix (donc le remords), celui de ce qu'il faut dire d'abord, en même temps, ou ensuite ou bien encore à côté.

\section{NOTES}

1. S. Boucheron, Parenthèses et double tiret : analyse linguistique de l'opération de décrochement typographique (1996). Thèse nouveau régime, sous la direction de Jacqueline Authier, Université de Paris III.

2. Cet essai graphique d'écorché du décroché se trouve, pour ainsi dire, corroboré par la parution du dernier roman de Claude Simon, Le Jardin des plantes dont les premières pages présentent ainsi un «montage » de différentes séquences placées dans plusieurs colonnes qui, se succédant ou s'indentant, rompent la linéarité nécessaire du langage.

3. Voir à ce titre, L. Dällenbach (1960). «Le tissu de mémoire », post-face à Claude Simon, La Route des Flandres, coll. « Double », Minuit, Paris.

4. Cité par B. Andrès (1992). Profils du personnage chez Claude Simon, Paris, Minuit, p. 11.

5. J. Authier (1993). «Jeux méta-énonciatifs avec le temps», dans H.Parret (sous la dir. de), Temps et Discours, Presses Universitaires de Louvain, coll. « La pensée philosophique », p. 87105.

6. Id. ibid., p. 102.

7. Id. ibid, p. 103.

8. Id. Ibid, p.103.

9. Id. ibid, p. 104.

10. Claude Simon, cité par J. Authier, op. cit., p.104.

11. Id. ibid, p. 104. 


\begin{abstract}
We set out to deal with the question of linearity through punctuation, especially through the effects of brackets. In the incipit of Claude Simon's Palace, it can be noticed that the linearity of language gets double. A first level, subjected to the scriptural norms of the nouveau roman, provides a hyperrealistic description of a pigeon ; a second level, brought about by brackets, reintroduces traces of subjectivity : novel characters, the modalizing subjectivity of a narrator and the thread of writing, which reappears at the heart of the text. This text is thus written along several lines, and makes it as if it was possible to avoid the language linearity which ordinarily constrains the writer.
\end{abstract}

\title{
KEYWORDS
}

Brackets, punctuation, text, linearity, subjectivity, doubling, typographical slide. 
